\title{
Preparation and comparative properties of membranes based on PANI and three inorganic fillers
}

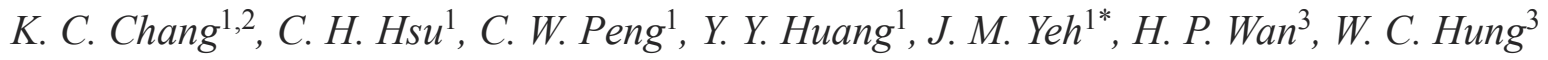 \\ ${ }^{1}$ Department of Chemistry, Center for Nanotechnology and Biomedical Technology at Chung-Yuan Christian University \\ (CYCU), Chung Li, 32023 Taiwan, Republic of China \\ ${ }^{2}$ R\&D Center for Membrane Technology, Chung-Yuan Christian University, Chung-Li, 32023 Taiwan, Republic of China \\ ${ }^{3}$ Green Energy and Environment Research Laboratories, Industrial Technology Research Institute, Room 308B, Building \\ 64, 195, Section 4, Chung Hsing Road, Chutung, 31040 Hsinchu, Taiwan
}

Received 14 August 2013; accepted in revised form 9 November 2013

\begin{abstract}
In this study, we compare the effects of aniline-modified mesoporous silica (AMS), raw silica (ARS) and nonmodified raw silica (NRS) particles on the physical properties of as-prepared polyaniline (PANI)-silica mesocomposites (PSM) and nanocomposites (PSN) and PANI-raw silica (PRSN) membranes. First, aniline-modified silica particles were synthesized by a conventional base-catalyzed sol-gel reaction of tetraethyl orthosilicate (TEOS) in the presence or absence of N-[3-(Trimethoxysilyl) propyl]aniline (PAPTMS). Subsequently, PSM, PSN and PRSN materials were prepared through in situ oxidation polymerization reaction of aniline monomer in the presence of AMS, ARS and NRS particles. It should be noted that all the properties of PSM membranes improved substantially from those of PSN and PRSN. For example, upon $3 \mathrm{wt} \%$ loading of AMS particles, 10, 25, 10, and 85\% increases in thermal stability, mechanical strength, surface hydrophilicity and gas permeability were observed for PSM membranes, respectively, as well as more than $45 \%$ reduction in the thermal conductivity.
\end{abstract}

Keywords: nanocomposites, polyaniline, silica, mesocomposite, membrane

\section{Introduction}

Organic-inorganic nanoparticle composite materials have gained substantial industrial and academic interest in recent years. Incorporating inorganic nanoparticles into organic polymers will improve the mechanical, thermal, optical and barrier properties. Commonly used inorganic additives include clay $[1,2], \mathrm{SiO}_{2}$ [3-5], carbon nanotubes [6, 7], and carbon nanofibers [8], among others.

Polyaniline (PANI) is a potential material for commercial applications owing to its facile synthesis, relatively low cost, environmental stability, good processability, and unique optical, electrical and electrochemical properties $[9,10]$. In recent decades, extremely promising applications have been reported for PANI in optical [11] and nanoelectronic devices [12], batteries [13], sensors [14], electrorheological materials $[15,16]$ and so on.

Mesoporous materials are a new class of nanoscale materials that possess large surface areas and have pore sizes that can be tuned within $2-10 \mathrm{~nm}$ in a very narrow distribution by varying the preparation conditions. Numerous potential applications of these materials are expected in the areas of catalysis, separation, adsorption and advanced materials. Recently, mesoporous silica-reinforced polymer composites have attracted considerable interest due to the possibility of improving the mechanical and thermal properties of polymers [17, 18]. Other interesting properties of these composites are, for example, low

\footnotetext{
${ }^{*}$ Corresponding author, e-mail: juiming@cycu.edu.tw

(C) BME-PT
} 
dielectric constants [19], low thermal expansion properties [20], and excellent friction and wear performances [21]. The incorporation of a guest monomer or polymer into the channels of mesoporous materials is a key factor contributing to the dispersion of additives in the polymer matrix and the enhancement of the properties of as-prepared composites.

There is a considerable amount of literature associated with the preparation and properties of polymersilica meso- and nanocomposite materials. However, there are fewer reports on the systematic comparative studies on their preparation and properties. In recent years, our group has reported the preparation of PMMA-silica [22] and PS-silica [23] meso- and nanocomposite membranes (thermoplastic polymer systems) and compared the incorporation of silica and mesoporous silica particles. The mesocomposite membranes exhibited enhanced mechanical strength, thermal stability, thermal and electric insulation, optical clarity, and surface hydrophobic properties in comparison to nanocomposite membranes. In general, comparative studies on the physical properties of conducting polymer-silica mesocomposites and their corresponding nanocomposite membranes have seldom been reported.

Therefore, in this paper, we present a systematic comparative study on the preparation and properties of as-prepared polyaniline (PANI)-silica mesocomposite (PSM), nanocomposite (PSN) and PANI-raw silica (PRSN) membranes. First, we synthesized aniline-modified mesoporous silica (AMS) and raw silica (ARS) particles by conventional base-catalyzed sol-gel reaction of TEOS in the presence of PAPTMS. Non-modified raw silica (NRS) particles were also synthesized by the base-catalyzed sol-gel reactions. The as-prepared particles were then characterized by Fourier transformation infrared (FTIR), ${ }^{13} \mathrm{C}$-nuclear magnetic resonance (NMR), and ${ }^{29} \mathrm{Si}$ NMR spectroscopies. Subsequently, meso- and nanocomposite materials were prepared by in situ oxidation polymerization of aniline in the presence of as-prepared AMS, ARS or NRS particles. The dispersibility of silica particles in the PANI matrix was further studied by transmission electron microscope (TEM). Gel permeation chromatography (GPC) was used for determining the molecular weights of as-prepared samples. The effect of material composition on the thermal stability, mechanical strength, thermal transport, surface and gas permeability properties of PSM, PSN and PRSN membranes were investigated by thermo-gravimetric analysis (TGA), dynamic mechanical analysis (DMA), the transient plane source (TPS) technique, contact angle measurement and gas permeability analysis (GPA) technique, respectively.

\section{Experimental}

\subsection{Materials and instrumentation}

Aniline (Fluka, Germany, 98.0\%) was used as a monomer and ammonium persulfate (APS, Fluka, Germany, $98.0 \%$ ) as an oxidant for the preparation of polyaniline. Tetraethyl orthosilicate (TEOS, Fluka, Germany, 98\%) and N-[3-(Trimethoxysilyl)propyl] aniline (PAPTMS, Aldrich, Germany) were used as a sol-gel precursor and silane coupling agent, respectively. D-(-)-Fructose (Aldrich, Germany, 99.0\%), ethanol (EtOH, Riedel-de Haën, Germany, 99.8\%), hydrochloric acid (HCl, Riedel-de Haën, Germany, $37 \%)$, ammonia solution $\left(\mathrm{NH}_{3}\right.$, Riedel-de Haën, Germany, 25\%), and N-methyl-2-pyrrolidinone (NMP, Tedia, USA, 99.0\%) were used without any further purification.

Brunauer-Emmett-Teller (BET, USA) characterizations were performed on a Micrometrics ASAP 2010 micropore analysis system at $-196^{\circ} \mathrm{C}$ using a nitrogen adsorption-desorption isotherm method. The samples were degassed at $120^{\circ} \mathrm{C}$ and $1 \mathrm{~Pa}$ overnight prior to the measurement. Pore sizes were obtained from the maximum of the pore-size distribution using a $\mathrm{BJH}$ model applied to the adsorption data. Both ${ }^{13} \mathrm{C}$ and ${ }^{29} \mathrm{Si}$ MAS solid-state NMR experiments were performed on a $400 \mathrm{MHz}$ Chemagnetics solid-state NMR spectrometer (BRUKER AVANCE 400, USA). ${ }^{13} \mathrm{C}$ MAS NMR spectra were obtained at $100.63 \mathrm{MHz}$ using a MAS frequency of $7 \mathrm{kHz}$ and applying $90^{\circ}$ pulses at $2.0 \mathrm{~s}$ pulse delays. To enhance the carbon sensitivity, cross-polarization $(\mathrm{CP})$ techniques were employed. ${ }^{29} \mathrm{Si}$ MAS NMR spectra were recorded at $79.49 \mathrm{MHz}$ in $5.0 \mathrm{~mm}$ zirconia sample rotors spinning at $7 \mathrm{kHz}$, applying $90^{\circ}$ pulses, $300 \mathrm{~s}$ pulse delays, and $5.0 \mathrm{~ms}$ contact time. FTIR spectra were obtained at a resolution of $4.0 \mathrm{~cm}^{-1}$ with a FTIR (JASCO FT/IR-4100, Japan) spectrophotometer at room temperature in a wavenumber range from 4000 to $400 \mathrm{~cm}^{-1}$. The microstructures of PSM and PSN membranes were imaged with a JEOL-200FX TEM (Japan). The samples for the TEM study were cut into 60-90 nm thick-sections with a diamond knife. The number-average and weight-average molecular 
weights of the polymers extracted from all composite samples and from the hybrid materials were determined on a Waters GPC-150CV (USA) equipped with a differential refractometer detector and a Styragel HT column using NMP as eluent and monodisperse polystyrenes as calibration standards. TGA was employed to record the thermal stability of the specimens. TGA scans were performed on a DuPont TA Q50 (USA) thermal analysis system in air atmosphere. The scan rate was $20^{\circ} \mathrm{C} / \mathrm{min}$ in a temperature range from 30 to $800^{\circ} \mathrm{C}$. DMA of the PSM and PSN membranes was performed in a temperature range from 40 to $300^{\circ} \mathrm{C}$ on a DuPont TAQ800 (USA) analyzer at a heating rate of $3^{\circ} \mathrm{C} / \mathrm{min}$ and at a frequency of $1 \mathrm{~Hz}$. The sample dimension was $10 \mathrm{~mm} \times 5 \mathrm{~mm}$. Thermal transport measurements employed a Hot Disk Thermal Constants Analyzer TPS (Taiwan) supplied by Hot Disk Inc. Contact angles of the samples were measured using a First Ten Angstroms FTA 125 (Germany) with a membrane thickness of $40 \mu \mathrm{m}$. A Yanagimoto Co., Ltd. gas permeability analyzer (model GTR 10, Japan) was employed to perform oxygen/nitrogen gas permeation experiments.

\subsection{Synthesis of aniline-modified raw silica (ARS) particles}

Aniline-modified silica (denoted as ARS) was prepared by a sol-gel reaction of TEOS and PAPTMS under base-catalyzed conditions, as shown in Fig- ure 1. The typical procedure is as follows: $300 \mathrm{~mL}$ ethanol, $5.2 \mathrm{~g}$ D-(-)Fructose and $4 \mathrm{~mL} 15 \mathrm{M} \mathrm{NH}_{3}$ solution were mixed and stirred for $10 \mathrm{~min}$ at room temperature. Simultaneously, a mixture of $5.2 \mathrm{~g}$ of TEOS and $1 \mathrm{~g}$ of PAPTMS was stirred in another container at room temperature. Then, this solution was slowly added to the first solution and stirred overnight. The mixture was centrifuged in ethanol five times to remove $\mathrm{NH} 3$, followed by drying in a vacuum oven. Non-modified raw silica (NRS) particles were also prepared as a reference without the addition of PAPTMS monomer, as shown in Figure 1.

\subsection{Synthesis of aniline-modified mesoporous silica (AMS) particles}

Aniline-modified mesoporous silica (denoted as AMS) was obtained by $\mathrm{NH}_{3}$-catalyzed sol-gel reaction of tetraethylorthosilicate (TEOS) and N-[3-(Trimethoxysilyl)propyl]aniline (PAPTMS) in the presence of non-surfactant template, D-(-)-Fructose, followed by the template removal by centrifugation, as shown in Figure 1. The procedure for the preparation of AMS in this work is as follows: $300 \mathrm{~mL}$ ethanol solution, $5.2 \mathrm{~g}$ D-(-)-Fructose and $4 \mathrm{~mL} 15 \mathrm{M}$ $\mathrm{NH}_{3}$ solution were mixed and stirred for $10 \mathrm{~min}$ at room temperature. Simultaneously, the mixture, of $5.2 \mathrm{~g}$ of TEOS and $1 \mathrm{~g}$ of PAPTMS was stirred in other container at room temperature. Then, this solution was slowly added into the first solution and stirred overnight. The mixture was centrifuged in

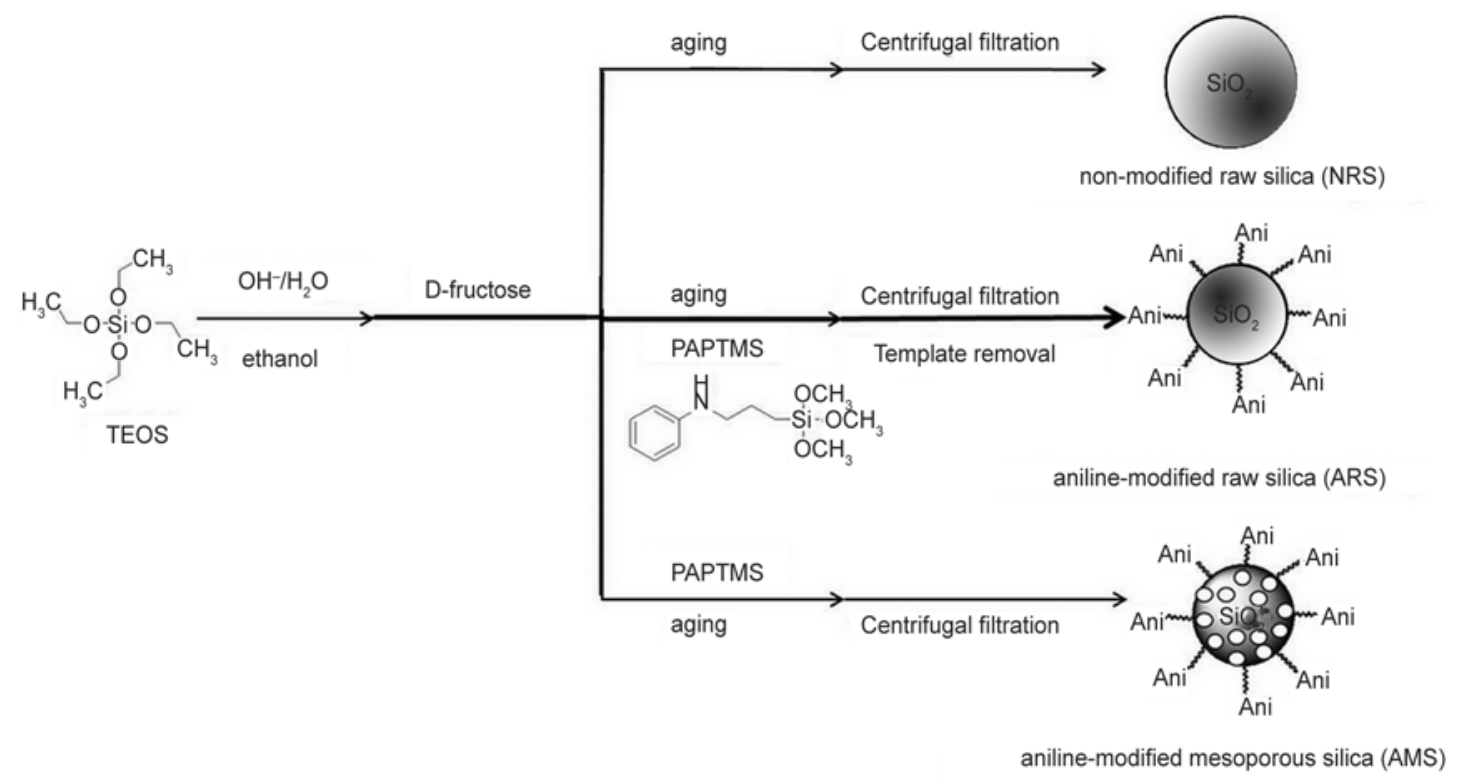

Figure 1. Synthesis of non-modified raw silica (NRS), aniline-modified raw silica (ARS) and aniline-modified mesoporous silica (AMS) particles 
ethanol five times to remove $\mathrm{NH}_{3}$ and in deionized water to remove D-(-)-Fructose, followed by drying in a vacuum oven.

\subsection{Preparation of PSM and PSN materials}

A typical procedure for the preparation of PSM and PSN materials was shown in Figure 2 and is given as follows: aniline monomer $(0.1 \mathrm{~mol})$ was added to $400 \mathrm{~mL}$ of $1.0 \mathrm{M} \mathrm{HCl}$, and various amounts of AMS $(1,3 \mathrm{wt} \%)$, ARS (3 wt \%) or NRS (3 wt $\%$ ) were fully dispersed in the solution under magnetic stirring. After adding APS $(0.025 \mathrm{~mol})$ in $20 \mathrm{~mL}$ of $1.0 \mathrm{M}$ $\mathrm{HCl}$, the solution was stirred for $6 \mathrm{~h}$ on an ice bath. The as-synthesized HCl-doped PSM or PSN precipitates were then filtered and dried under dynamic vacuum at $40^{\circ} \mathrm{C}$ for $2 \mathrm{~d}$. The final PSM or PSN materials were obtained in a base form by immersing them into $400 \mathrm{~mL}$ of $1.0 \mathrm{M} \mathrm{NH}_{4} \mathrm{OH}$ under magnetic stirring for $4 \mathrm{~h}$ at room temperature, followed by the filtration and drying under vacuum at $40^{\circ} \mathrm{C}$ for $2 \mathrm{~d}$.

\subsection{Preparation of composite membranes}

$0.2 \mathrm{~g}$ sample of PSM or PSN material in a base form was dissolved in $10 \mathrm{~mL}$ of NMP under magnetic stirring at room temperature for $1 \mathrm{~d}$. The solution was cast onto a substrate (e.g., glass), and the solvent was evaporated in an oven for $1 \mathrm{~d}$. The sample-coated glass substrate was then immersed in distilled water to yield PSM and PSN membranes. The membrane thickness was about $45 \mu \mathrm{m}$.

\section{Results and discussion}

Aniline-modified mesoporous silica (AMS) nanoscale particles with a wormhole framework structure were prepared by an energy-saving and environmentally friendly non-surfactant template route, developed by Wei et al. [24]. First, a TEOS/PAPTMS feed ratio of $8 / 2$ (i.e., AMS82) and an optically active D-(-)-Fructose non-surfactant as a template were used in the conventional base-catalyzed sol-gel reaction, followed by a template removal through

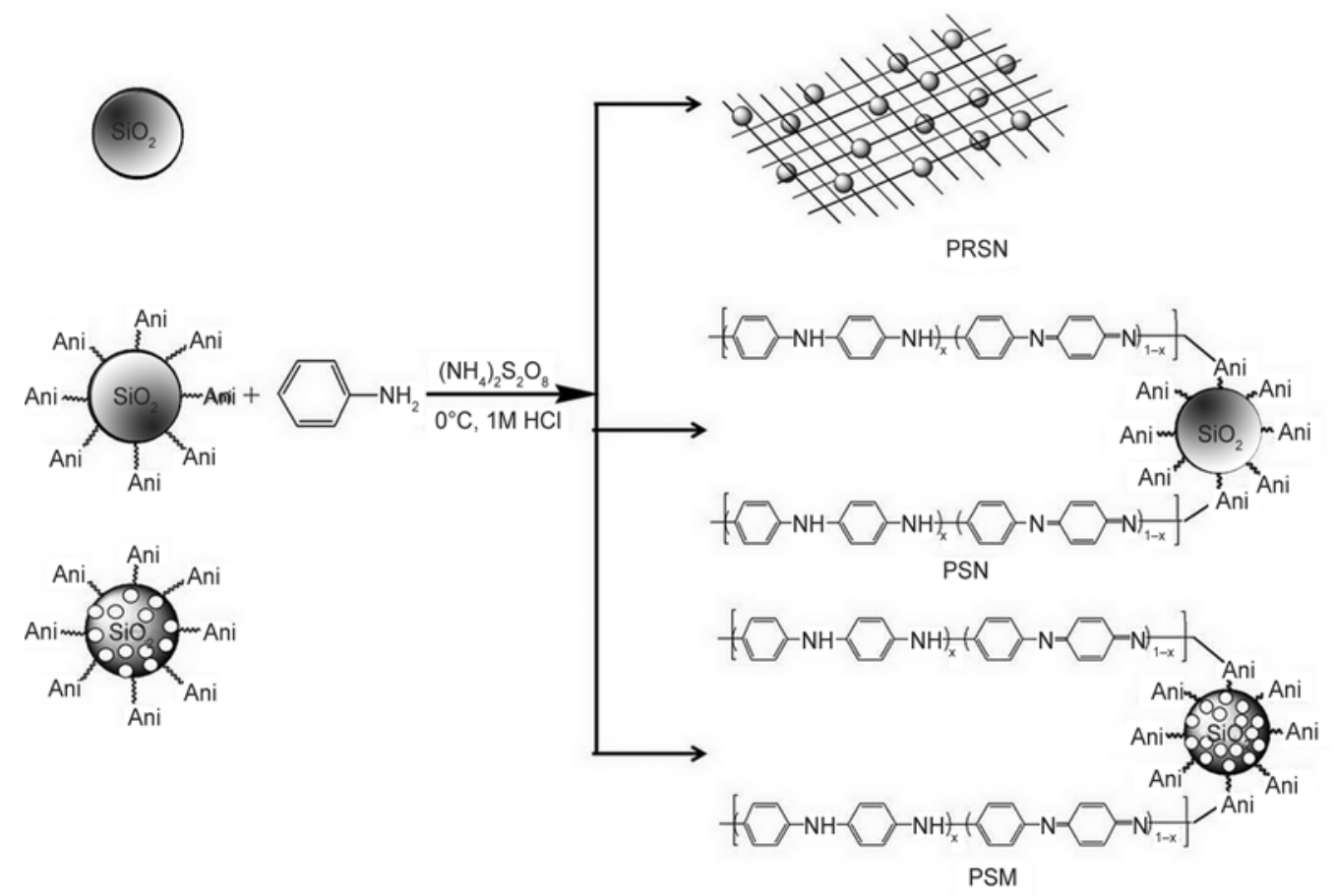

Figure 2. Preparation of PANI-raw silica (PRSN), and PANI-silica mesocomposite (PSM) and nanocomposite (PSN)

Table 1. Physical properties of nanoscale aniline-modified mesoporous and raw silica particles and PSM materials

\begin{tabular}{|l|c|c|c|c|c|c|}
\hline \multicolumn{1}{|c|}{ Sample code } & $\begin{array}{c}\text { TEOS } \\
{[\mathbf{m o l}]}\end{array}$ & $\begin{array}{c}\text { PAPTMS } \\
{[\mathbf{m o l}]}\end{array}$ & $\begin{array}{c}\text { D-Fructose } \\
{[\mathbf{w t} \mathbf{m}]}\end{array}$ & $\begin{array}{c}\mathbf{D}_{\mathbf{p}} \\
{[\mathbf{n m}]^{\mathbf{a}}}\end{array}$ & $\begin{array}{c}\mathbf{V}_{\mathbf{t}} \\
\left.\mathbf{c}^{\mathbf{3}} \mathbf{\mathbf { g }}\right]^{\mathbf{b}}\end{array}$ & $\begin{array}{c}\mathbf{S}_{\text {BET }} \\
{\left[\mathbf{m}^{\mathbf{2}} / \mathbf{g}\right]^{\mathbf{c}}}\end{array}$ \\
\hline RS & 0.0254 & - & - & - & 0.03 & 14.1 \\
\hline ARS82 & 0.0250 & 0.0004 & - & - & 0.03 & 8.2 \\
\hline MS & 0.0254 & - & 2 & 3.3 & 0.70 & 587.0 \\
\hline AMS82 & 0.0250 & 0.0004 & 2 & 3.4 & 0.50 & 506.0 \\
\hline PSM3 & 0.0250 & 0.0004 & 2 & 12.6 & 0.20 & 51.4 \\
\hline
\end{tabular}

${ }^{\mathrm{a}}$ Pore diameter.

${ }^{\mathrm{b}}$ Total pore volume calculated from $P / P_{0}=0.99$.

${ }^{\mathrm{c}}$ BET surface area. 
Soxhlet extraction. The aniline-modified raw silica (ARS) and non-modified raw silica (NRS) nanoscale particles were also prepared as references for systematic comparative studies. Specific surface area, ABET, determined from the linear part of BET plot, and pore size obtained from the maximum of pore size distribution using a BJH model applied to the adsorption data are summarized in Table 1. Subsequently, the selected amount of as-prepared AMS82/ ARS82/NRS82 particles was reacted with aniline monomer by free radical solution polymerization in toluene with APS initiator to give a series of polymer-silica meso- or nanocomposite materials.

\subsection{Characterization}

\subsubsection{Microstructure of nanoscale silica particles and corresponding PANI-silica meso- and nanocomposite membranes}

BET surface areas and pore volumes of the samples were determined by $\mathrm{N}_{2}$ adsorption-desorption isotherm measurements [25]. The isotherms of silica materials are shown in Figure 3. The aniline-modified silica particles showed relatively small BET surface areas and pore volumes compared to the non-modified silica particles (i.e., MS and RS), as summarized in Table 1. Moreover, MS particles prepared at $2 \mathrm{wt} \% \mathrm{D}-(-)$-Fructose template concentration exhibited type IV isotherms with type $\mathrm{H}_{2}$ hysteresis loops, showing one capillary adsorption at partial pressures $\left(P / P_{0}\right)$ of $0.4-0.8$ (Figure $3 a$ ), and are attributed to the mesopores [26]. The characteristic feature of the isotherms above is the hysteresis loop, which is associated with the capillary condensation taking place in the mesopores [27, 28] and the limiting uptake at high partial pressures. The initial part of the MS (without PAPTMS) iso- therm was attributed to monolayer-multilayer adsorption, as it follows the same path as the non-porous adsorbent with the same surface area. The characteristics of the remaining two isotherms are the hysteresis loops widely distributed to the lower partial pressure $\left(P / P_{0}\right)$ range of $0.01-0.8$. The samples with up to $20 \%$ PAPTMS show a steep increase in the adsorption at $P / P_{0}$ of $0.4-0.8$, indicating that the obtained materials possess large mesopores and narrow pore-size distribution similar to the pure silica, as the high amount of large functional group silanes occupy larger pore volume. On the other hand, samples containing 20\% PAPTMS have apparently lower absorption volumes and narrower pore diameter. Moreover, Barrett-Joyner-Halenda (BJH) pore diameter [29], BET surface area, and pore volume of AMS decreased with increasing aniline content, as shown in Figure $3 \mathrm{~b}$ and Table 1. These results were attributed to the occupation of the pore surface by large organic molecules, as well as to the perturbation of aniline groups during the silicate condensation process [26]. Further compare the pore volume of AMS particles and PSM3 materials, the pore volume of PSM3 is reduced to 0.2 from $0.5 \mathrm{~cm}^{3} / \mathrm{g}$ for AMS particles as a consequence of pore filling by PANI [30].

\subsubsection{Spectroscopic studies of AMS82/ARS82/NRS82 particles}

In this study, the as-prepared AMS particles were synthesized by base-catalyzed sol-gel reaction of TEOS in the presence of PAPTMS, and subsequently characterized by FTIR, ${ }^{13} \mathrm{C}-\mathrm{NMR}$ and ${ }^{29} \mathrm{Si}-$ NMR spectroscopies. Figure 4 shows the representative FTIR spectra of D-(-)-Fructose and the asprepared NRS, ARS and AMS particles. For example,

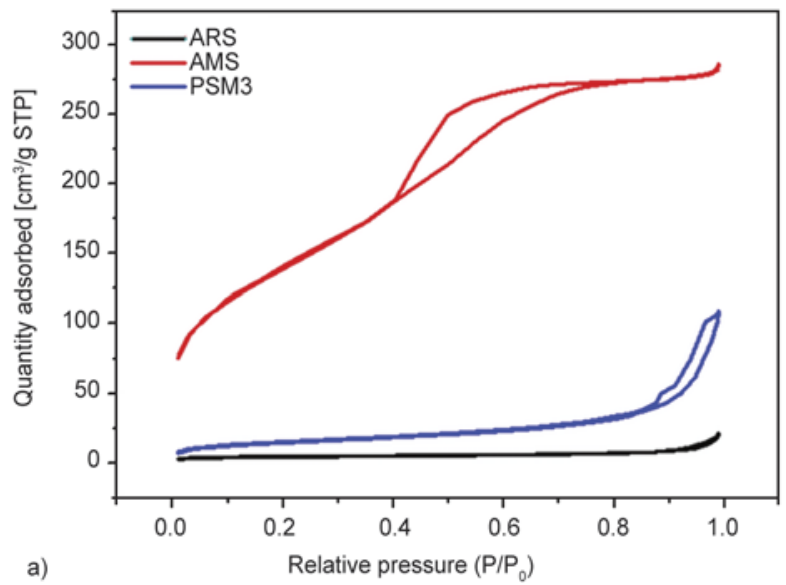

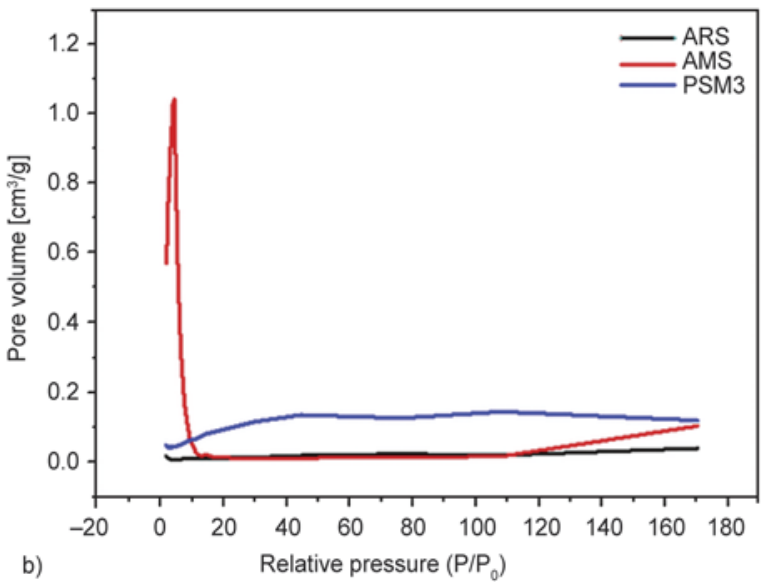

Figure 3. (a) $\mathrm{N}_{2}$ adsorption/desorption isotherms and (b) pore size distributions of ARS, AMS particles and PSM3 materials 


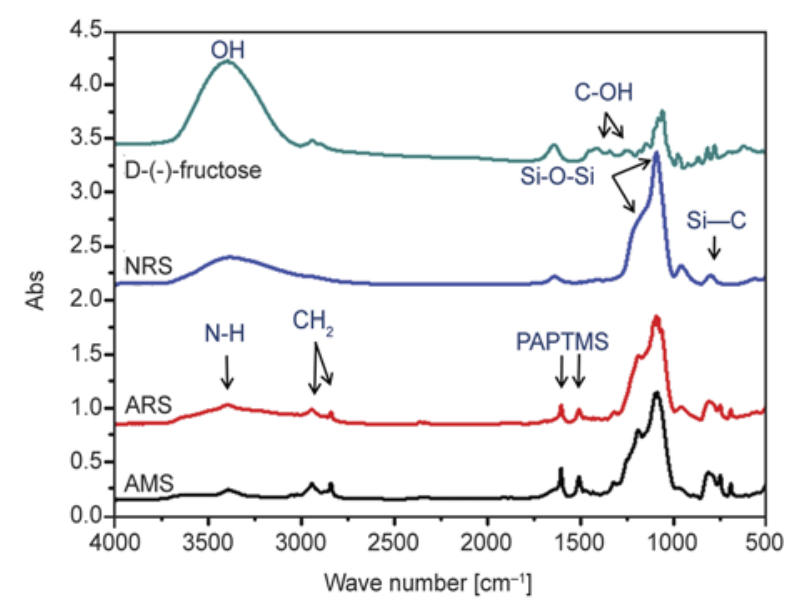

Figure 4. FTIR absorption spectra of D-(-)-Fructose, and NRS, ARS and AMS particles

the spectral bands characteristic of the aniline group of PAPTMS appearing at 1607 and $1505 \mathrm{~cm}^{-1}$ were assigned to the stretching of $\mathrm{C}=\mathrm{N}$ and $\mathrm{C}=\mathrm{C}$, corresponding to quinone and benzene ring stretching deformations, respectively. The positions of the absorption peaks are similar to those reported in refs. [31-34]. The presence of organic groups in the materials after the hydrolysis and condensation was confirmed by solid-state NMR spectroscopy. Figure 5 shows the solid-state ${ }^{13} \mathrm{C}$ CP MAS NMR spectra of AMS particles, where the AMS particles exhibit additional resonance peaks at chemical shifts $\delta=8.41,21.15,46.10,117.14,129.35$ and $146.43 \mathrm{ppm}$. These peaks are attributed to the different carbon environments in the organosilane, denoted as groups 1-3 and 4-6 [35], indicating the incorporation of aniline functional groups. Moreover, solid-state ${ }^{29} \mathrm{Si}$ MAS NMR provided information about the silicon environment. The ${ }^{29} \mathrm{Si}$ MAS NMR spectra of AMS particles are presented in Figure 6. Two resonance peaks arising from the silicon environments of $\mathrm{Si}(\mathrm{OSi})_{4}\left(\mathrm{Q}^{4}, \delta=-113.79 \mathrm{ppm}\right)$

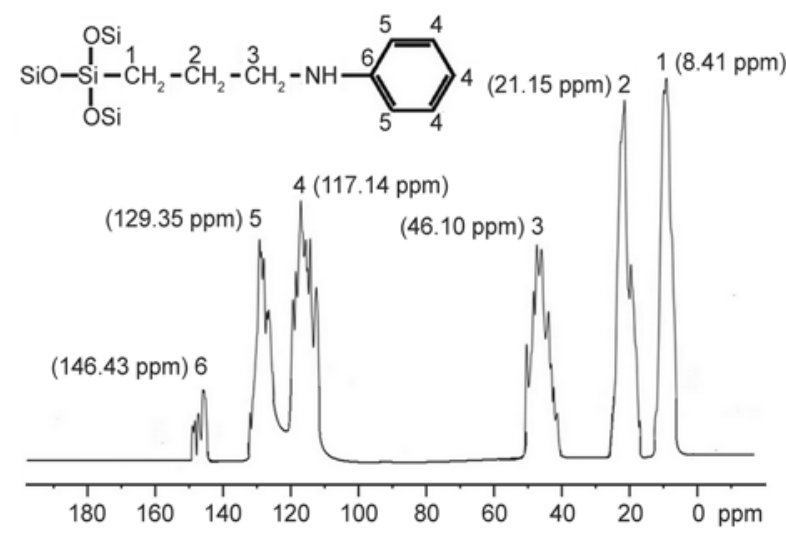

Figure 5. ${ }^{13} \mathrm{C}$ solid-state NMR spectra of AMS particles

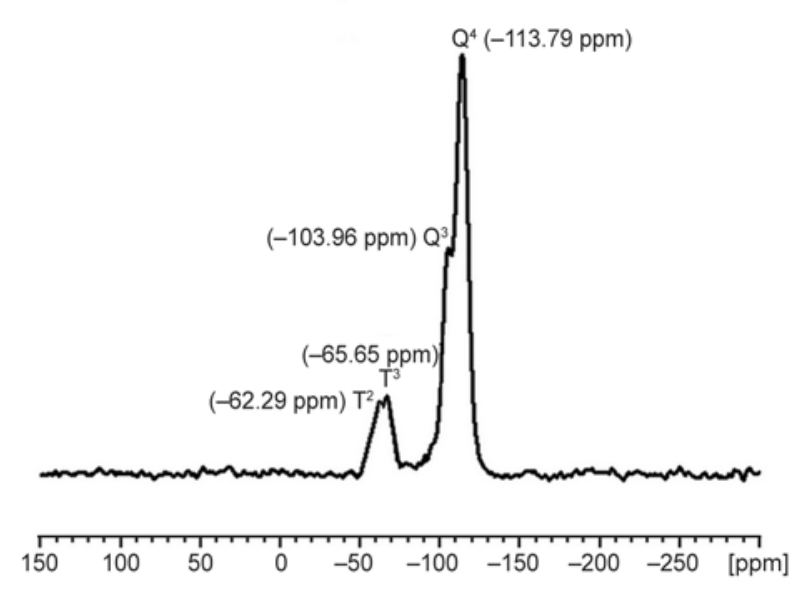

Figure 6. ${ }^{29} \mathrm{Si}$ solid-state NMR spectra of AMS particles and $\mathrm{HOSi}(\mathrm{OSi})_{3}\left(\mathrm{Q}^{3}, \delta=-103.96 \mathrm{ppm}\right)$ can be observed [35]. In addition, the AMS particles display two more resonance peaks at $\delta=-65.65 \mathrm{ppm}$, assigned to $\mathrm{R}-\mathrm{Si}(\mathrm{OSi})_{3}\left(\mathrm{~T}^{3}\right)$, and at $\delta=-62.29 \mathrm{ppm}$, attributed to $\mathrm{R}-\mathrm{Si}(\mathrm{OH})(\mathrm{OSi})_{2}\left(\mathrm{~T}^{2}\right)$ [35]. These two peaks arise from the silicon atoms at different environments in the organosilane PAPTMS.

\subsubsection{FTIR spectra of PANI-silica mesocomposites}

The FTIR spectra of AMS particles, PANI and PSM3 are shown in Figure 7. The FTIR spectrum of AMS particles shows the absorption bands at $1096 \mathrm{~cm}^{-1}$ (asymmetric $\mathrm{Si}-\mathrm{O}-\mathrm{Si}$ stretch), $956 \mathrm{~cm}^{-1}$ ( $\mathrm{Si}-\mathrm{OH}$ vibrations) and $816 \mathrm{~cm}^{-1}$ (symmetric $\mathrm{Si}-\mathrm{O}-\mathrm{Si}$ stretch). In the case of PANI, the main peaks at 1588 and $1483 \mathrm{~cm}^{-1}$ can be assigned to the stretching vibrations of quinone and benzene rings, respectively. The peaks at 1282 correspond to the $\mathrm{C}-\mathrm{N}$ stretching vibration. The in-plane bending of $\mathrm{C}-\mathrm{H}$ is reflected in the $1155 \mathrm{~cm}^{-1}$ peak. The peak at $810 \mathrm{~cm}^{-1}$ is attributed to the out-of-plane bending of $\mathrm{C}-\mathrm{H}$. The FTIR spectrum of PSM3 implies that the mesocomposites contained the characteristic peaks of AMS and PANI. As expected, when com-

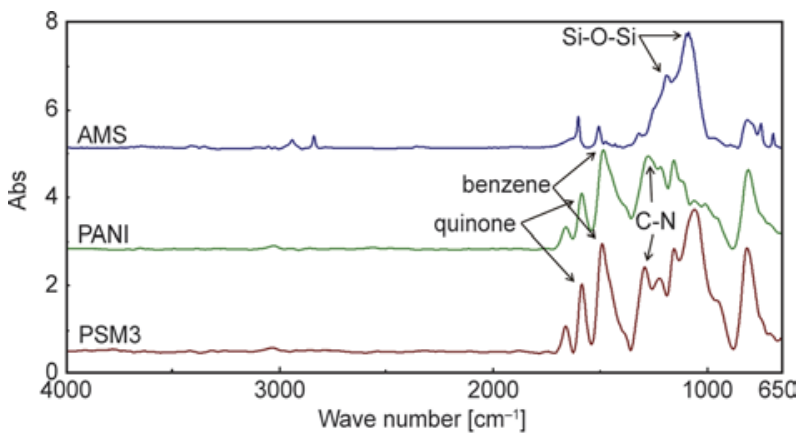

Figure 7. FTIR spectra of AMS particles, PANI and PSM3 
paring the spectra of PANI and the mesocomposites, the absorption bands all shift to higher wavenumbers in the mesocomposites. These phenomena indicate that some interaction of PANI with the host $[36,37]$.

\subsubsection{Morphological observations of silica particles and corresponding PANI-silica meso- and nanocomposite membranes}

The morphology of the silica particles and their corresponding PANI-silica meso- and nanocomposites can be directly confirmed by TEM. Figure 8 shows the TEM images of the silica and mesoporous silica particles synthesized in this work. The NRS (Figure 8a), ARS (Figure 8b) and AMS particles (Figure $8 \mathrm{c}$ ) have the sizes of about 250 and $300 \mathrm{~nm}$, respectively, and they are spherical. The mesoporous silica nanospheres have cylindrical channels and highly ordered mesoporous structure (Figure $8 \mathrm{c}$ ). Figure 9 shows the TEM images of PANI/ silica meso- and nanocomposites. With the exception of the composites made from ARS and AMS particles (Figure 9b-c), the images exhibit silica and mesoporous silica nanoparticles with sizes ranging from about 200 to $300 \mathrm{~nm}$, relatively welldispersed in the polymer matrix. However, the TEM image of PRSN3 obviously shows the aggregation of silica particle into clusters, indicating the poor dispersibility of silica particles in the PANI matrix in contrast to aniline-modified silica and mesoporous silica nanoparticles, as shown in Figure 9a.

\subsubsection{Molecular weights and polydispersity of composite membranes}

Molecular weights of neat polymer and various composites were obtained by GPC analyses using THF as an eluent. All the GPC traces of samples exhibited a single peak, represented by the molecular weight values summarized in Table 2 . The molecular weights of all composite materials were lower than that of neat PANI, indicating structurally restricted polymerization in the neighboring region of silica particles (e.g., NRS82/ARS82/AMS82) and/or silica-oligomer interactions, such as adsorption, during the polymerization reaction. For example, the values of weight-average molecular weight $\left(\bar{M}_{\mathrm{w}}\right)$, number-average molecular weight $\left(\bar{M}_{\mathrm{n}}\right)$ and polydispersity (PDI) of neat PANI are 25600 , 12100 and 2.12, respectively. Upon the incorporation of $3 \mathrm{wt} \%$ of AMS82 particles into PANI (i.e., PMS3) by in situ solution polymerization, these values showed a clearly decreasing trend to 22000 , 10,500 and 2.09, respectively. Moreover, the decreasing trend of molecular weights and PDI of the nanocomposite system is very close to that of mesocomposite system, and thus, the reactive channel aniline groups of nanoscale mesoporous silica show a slight effect on the molecular weights and molecu-

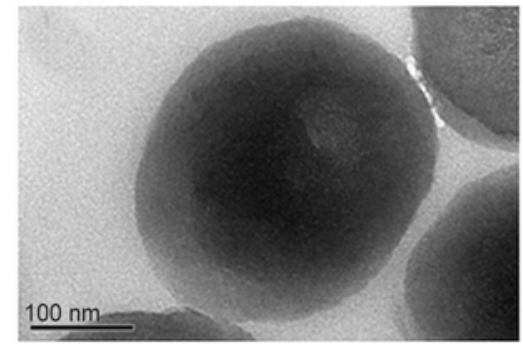

a)

.

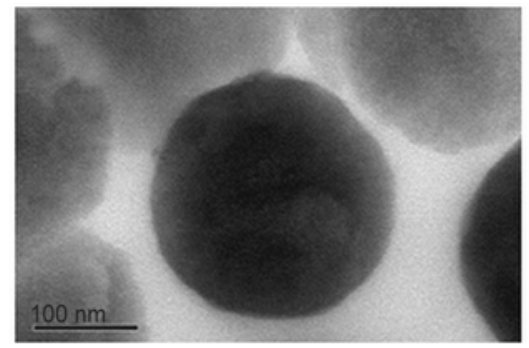

b)

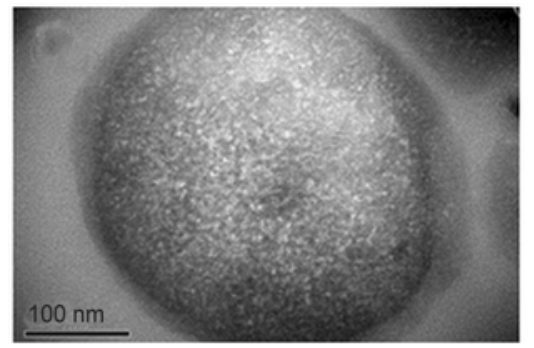

c)

Figure 8. TEM images of (a) NRS, (b) ARS, and (c) AMS particles

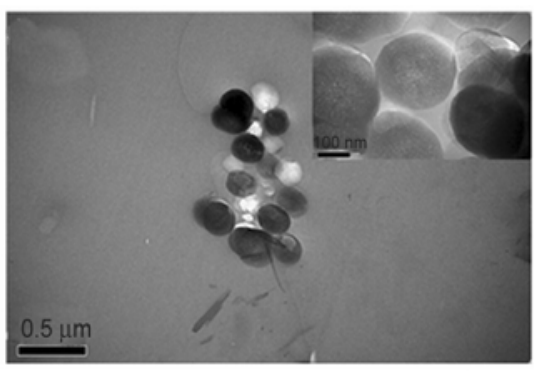

a)

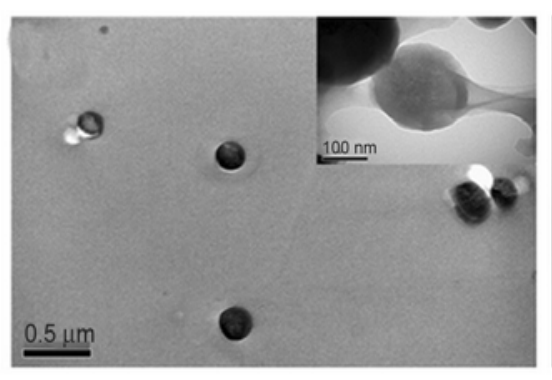

b)

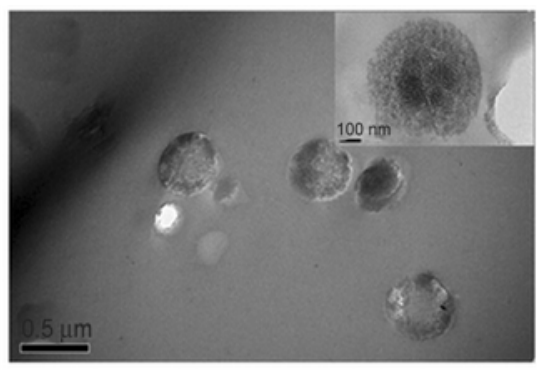

c)

Figure 9. TEM images of (a) PRSN3, (b) PSN3 and (c) PSM3. Illustration is the large magnification of images (50 k). 
Table 2. Molecular weight, thermal, mechanical, surface hydrophobic and gas permeability properties of PANI and composite membranes

\begin{tabular}{|c|c|c|c|c|c|c|c|c|c|c|c|c|c|c|}
\hline \multirow{2}{*}{$\begin{array}{c}\text { Compo } \\
\text { und } \\
\text { code }\end{array}$} & \multicolumn{4}{|c|}{$\begin{array}{c}\text { Feed composition } \\
\text { [wt } \%]\end{array}$} & \multicolumn{3}{|c|}{ Molecular weight } & \multicolumn{2}{|c|}{$\begin{array}{l}\text { Thermal } \\
\text { properties }\end{array}$} & \multirow{2}{*}{\begin{tabular}{|c}
$\begin{array}{c}\text { Mechanical } \\
\text { properties }\end{array}$ \\
$\mathbf{E}^{\prime}$ \\
{$\left[\mathrm{MPa}^{\mathrm{d}}\right.$}
\end{tabular}} & \multirow[t]{2}{*}{$\begin{array}{c}\text { Contact } \\
\text { angle } \\
{\left[^{\circ}\right]}\end{array}$} & \multicolumn{2}{|c|}{$\begin{array}{l}\text { Permeability } \\
\text { [barrer] }^{\mathrm{e}}\end{array}$} & \multirow{2}{*}{$\begin{array}{c}\begin{array}{c}\text { Perms- } \\
\text { electivity }\end{array} \\
\alpha_{\mathrm{O}_{2} / \mathrm{N}_{2}}\end{array}$} \\
\hline & PANI & RS & ARS & AMS & $\overline{\mathbf{M}}_{\mathrm{w}}$ & $\overline{\mathbf{M}}_{\mathbf{n}}$ & PDI $^{\mathbf{a}}$ & $\begin{array}{c}\mathbf{T}_{\mathbf{d}} \\
\left.{ }^{\circ} \mathbf{C}\right]^{b}\end{array}$ & $\begin{array}{c}\mathbf{k} \\
{[\mathbf{W} /(\mathbf{m} \cdot \mathbf{K})]^{\mathbf{c}}}\end{array}$ & & & $\mathbf{P}_{\mathrm{O}_{2}}$ & $\mathbf{P}_{\mathrm{N}_{2}}$ & \\
\hline PANI & 100 & - & - & - & 25600 & 12100 & 2.12 & 440.70 & 0.0933 & 2610 & $90 \pm 2$ & 0.1972 & 0.0305 & 6.4585 \\
\hline PRSN3 & 97 & 3 & - & - & 21200 & 11200 & 1.90 & 460.60 & 0.1504 & 2720 & $93 \pm 2$ & 0.1889 & 0.0292 & 6.4582 \\
\hline PSN3 & 97 & - & 3 & - & 23200 & 10500 & 2.22 & 470.50 & 0.1153 & 3020 & $92 \pm 2$ & 0.1892 & 0.0293 & 6.4581 \\
\hline PSM1 & 99 & - & - & 1 & 25000 & 13100 & 1.91 & 480.30 & 0.0618 & 3240 & $85 \pm 2$ & 0.3608 & 0.0567 & 6.3616 \\
\hline PSM3 & 97 & - & - & 3 & 22000 & 10500 & 2.09 & 490.90 & 0.0498 & 3320 & $80 \pm 2$ & 0.3696 & 0.0602 & 6.1407 \\
\hline
\end{tabular}

${ }^{\mathrm{a} P D I}=\mathrm{M}_{\mathrm{w}} / \mathrm{M}_{\mathrm{n}}$.

${ }^{\mathrm{b}}$ As measured by TGA.

${ }^{c}$ As measured by Hot Disk.

${ }^{\mathrm{d} A s}$ measured by DMA.

${ }^{\mathrm{e}} \mathrm{At} 35^{\circ} \mathrm{C}, 1$ barrer $=10^{-10} \mathrm{~cm}^{3}(\mathrm{STP}) \mathrm{cm} / \mathrm{cm}^{2} \mathrm{~s} \mathrm{cmHg}$.

lar weight distributions in all as-prepared composite materials.

\subsection{Thermal properties}

\subsubsection{Thermogravimetric analysis}

Figure 10 shows the thermal stabilities of the composite membranes determined by a thermogravimetric analyzer. Upon the addition of silica particles, all the composite membranes had higher thermal decomposition $\left(T_{\mathrm{d}}\right)$ temperatures than PANI membrane. Typically, the $T_{\mathrm{d}}$ values corresponding to the $10 \mathrm{wt} \%$ weight loss of the composite membranes shifted to higher temperature range with increasing mesoporous silica content, which confirms the enhanced thermal stability of the composite membranes, as shown in Figure 10 and Table 2. Moreover, with the same amount of silica particles, the PSM3 membrane showed higher $T_{\mathrm{d}}$ than PSN3 and PRSN3 membranes. Generally, PANIsilica membranes are expected to exhibit better thermal stability upon the incorporation of silica particles. The obvious differences in the increase in $T_{\mathrm{d}}\left(50.2^{\circ} \mathrm{C}\right.$ increase for PSM 3 membrane, $29.8^{\circ} \mathrm{C}$ for PSN3 membrane and $19.9^{\circ} \mathrm{C}$ for PRSN3 membrane) may be attributed to the following: each nano-

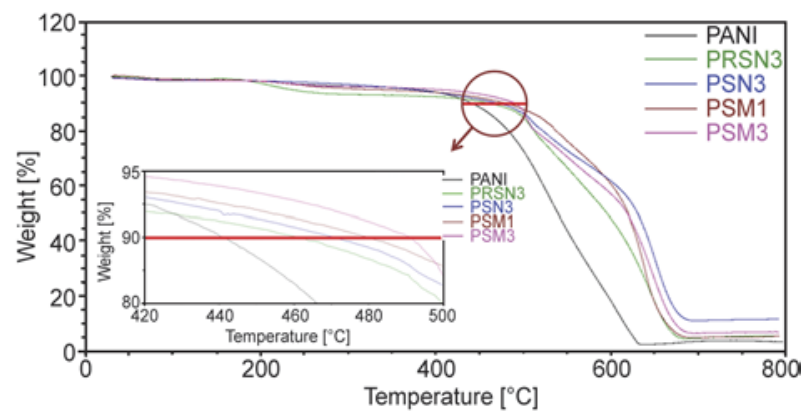

Figure 10. Thermal stabilities of the composite membranes scale AMS82 nanoparticle has abundant aniline groups on the surface and on the surface of internal pore channels. Therefore, the improvement in the thermal stability of the PSM membrane can be ascribed both to the good thermal stability of AMS and to the multilinkage that occurs between the PANI chains and AMS. Hence PANI chains not only bond to the surface of the AMS particles but also penetrate through the pore channels and are tethered to the channel surface.

Moreover, the characteristic yield for all the PSM, PSN and PRSN membranes increases with respect to the AMS82 weight percentage. This may be due to the PANI main chains on the AMS surface and inside the AMS pores, as well as due to the fact that ARS and NRS surfaces are more difficult to decompose than PANI.

\subsubsection{Thermal transport properties}

In this study, the thermal transport properties of PSM, PSN and PRSN membranes measured by TPS technique are shown in Figure 11 and Table 2. For

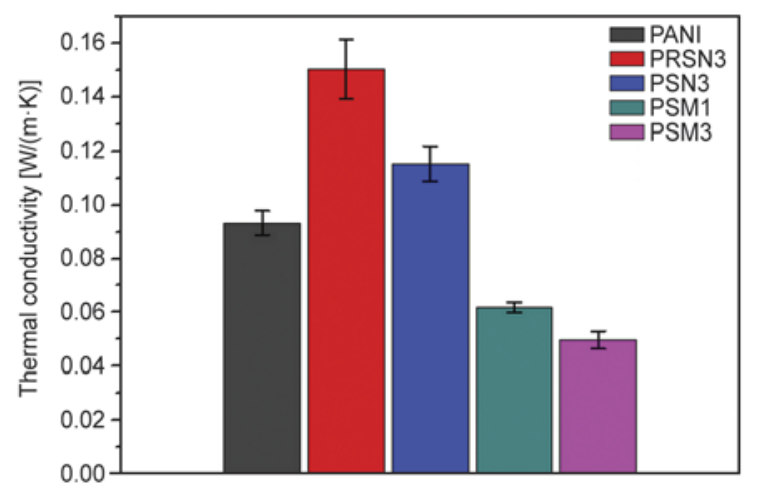

Figure 11. Thermal transport properties of the composite membranes 
PSN and PRSN membranes, the incorporation of ARS and NRS particles in the PANI matrix revealed an obvious increase in thermal conductivity. For example, PANI exhibited thermal conductivity $(k$, $\mathrm{W} /(\mathrm{m} \cdot \mathrm{K})$ ) of 0.0933 [38], which was lower than that of PSN and PRNS membranes. It should be noted that the $k$ value of the PSM membrane containing $3 \mathrm{wt} \%$ of AMS particles was clearly lower than those of PSN3, PRSN3, and PANI membranes. This is probably attributed to the existence of pores/ voids in the multilinkages between the PANI chains and the nanoscale internal channels of AMS particles, which may incorporate a relatively large amount of air $(k=0.029$ at $R T)$ [39] into the mesocomposite membranes, leading to the obvious decrease in the thermal conductivity [22].

\subsection{Mechanical properties}

To investigate the effect of silica and mesoporous silica on the mechanical properties of PSM, PSN and PRSN membranes, the composite membranes were studied by dynamic mechanical analysis (DMA). The storage modulus $\left(E^{\prime}\right)$ curves for the composite membranes over the temperature range from 40 to $300^{\circ} \mathrm{C}$ are shown in Figure 12, and the results are listed in Table 2 . It can be clearly seen that the incorporation of silica increased the $E^{\prime}$. Near room temperature, the PANI membrane has lower $E^{\prime}(2610 \mathrm{MPa})$ than the composite membranes $(>2720 \mathrm{MPa})$. With the same amount of silica particles, the membrane with incorporated mesoporous silica nanospheres (PSM3 membrane) shows the highest $E^{\prime}$ value compared with those with silica nanoparticles (PSN3 and PRSN3 membranes). The obvious difference in the mechanical strength enhancement of the membranes (27\% for PSM3 vs.

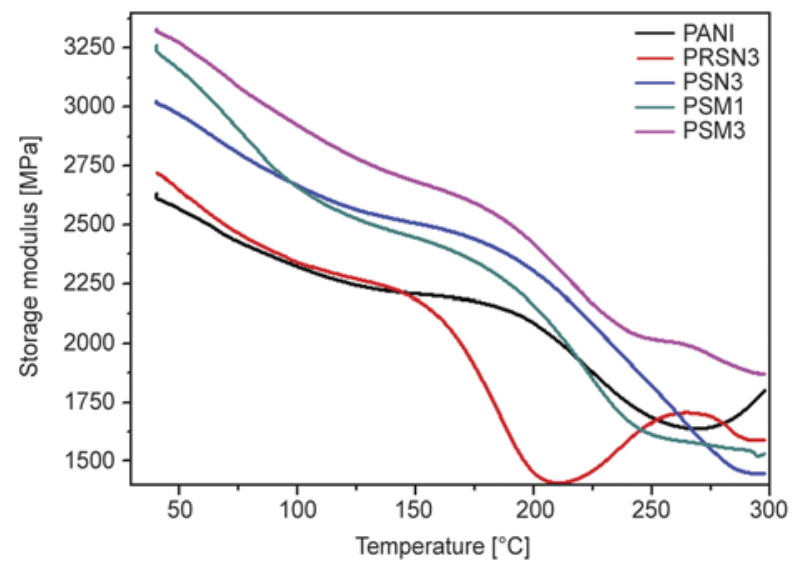

Figure 12. Mechanical properties of the composite membranes
$16 \%$ for PSM3 and 4\% for PRSM3 with AMS 82 , ARS82, and NRS82 particles in PANI, respectively) indicates that the reactive channel aniline groups of nanoscale mesoporous silica do show significant enhancement of the mechanical strength of as-prepared composites.

\subsection{Surface properties}

To investigate the surface properties of the membranes, contact angle tests were carried out, and the results are given in Table 2. The measured contact angle of pure PANI was $90^{\circ}$. The contact angle of the composite membranes decreased with increasing mesoporous silica content. This trend is due to the relative hydrophilicity of mesoporous silica particles. With the same silica particle content, PSM3 membrane exhibited lower contact angle than PSN3 and PRSN3 membranes. This behavior was attributed to the different properties of silica particles, such as hydrophilicity and particle size.

\subsection{Gas permeability properties}

For the comparative studies on the gas permeability of PSM, PSN and PRSN membranes, gas permeability analysis (GPA) technique was used for measuring the permeability of gases such as $\mathrm{O}_{2}$ and $\mathrm{N}_{2}$. The gas permeability of various membranes is shown in Figure 13, and the results are listed in Table 2. The results show that when mesoporous silica particles were added, the permeability of the composite membranes showed an obvious increase from that of PANI membrane and increased with increasing mesoporous silica content. At the same silica loading, the permeability of PSM3 membrane for $\mathrm{O}_{2}$ and $\mathrm{N}_{2}$ was higher than those of PSN3 and PRSN3 membranes.

The selectivity of gas pairs $\left(\mathrm{O}_{2} / \mathrm{N}_{2}\right)$ are also given in Table 2. In Table 2, the selectivity of the composite membranes shows a slight decrease over that of the

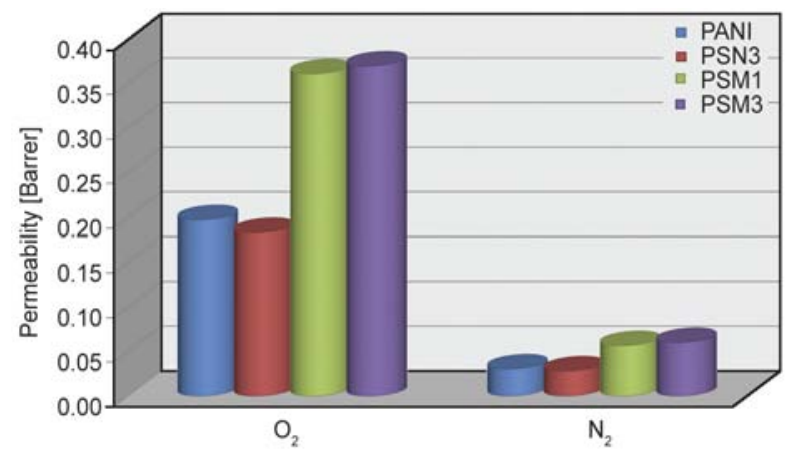

Figure 13. Gas permeabilities of the composite membranes 
PANI membrane, as expected. This phenomenon could be attributed to the fact that some of the gas penetrants bypassed the silica particles completely in the MMMs because of the presence of voids, resulting in blocked passage through the silica particles.

\section{Conclusions}

In this study, conductive PSM, PSN and PRSN membranes have been prepared successfully. The dispersibility of silica particles in PANI matrix was observed by TEM. GPC was used for determining the molecular weights of as-prepared samples. The effect of material composition on the mechanical strength, thermal stability, thermal transport, surface and gas permeability properties of PSM, PSN and PRSN membranes were investigated by TGA, TPS technique, DMA, contact angle measurement, and GPA, respectively. It should be noted that at the same silica loading, PSM membranes exhibited better thermal stability (e.g., higher $T_{\mathrm{d}}$ ), thermal insulation property (e.g., lower $k$ ), and mechanical strength (e.g., higher $E^{\prime}$ ), as well as increased surface hydrophility (e.g., higher wettability of water droplets) and gas permeability as compared with those of PSN and PRSN membranes. The obvious differences in the physical properties of all composite membranes may be attributed to the presence of reactive channel aniline groups and unoccupied channel voids/ pores in the nanoscale silica-based mesoporous materials.

\section{Acknowledgements}

We gratefully acknowledge the financial support of the Ministry of Education (MOE), Taiwan, R.O.C., NSC 1012113-M-033-005-MY1, the Center-of-Excellence (COE) Program on Membrane Technology from MOE, R.O.C., the Department of Chemistry at Chung Yuan Christian University (CYCU) (CYCU-01RD-RA002-11235), the Center for Nanotechnology and Biomedical Technology at CYCU, and the Bureau of Energy, Ministry of Economic Affairs (MOEA), Taiwan, R.O.C., for its financial support under Contract 102-D0106.

\section{References}

[1] Schütz M. R., Kalo H., Lunkenbein T., Gröschel A. H., Müller A. H. E., Wilkie C. A., Breu J.: Shear stiff, surface modified, mica-like nanoplatelets: A novel filler for polymer nanocomposites. Journal of Materials Chemistry, 21, 12110-12116 (2011).

DOI: $10.1039 / \mathrm{C} 1 \mathrm{JM} 11443 \mathrm{C}$
[2] Nishida T., Obayashi A., Haraguchi K., Shibayama M.: Stress relaxation and hysteresis of nanocomposite gel investigated by SAXS and SANS measurement. Polymer, 53, 4533-4538 (2012).

DOI: $10.1016 /$ j.polymer.2012.07.038

[3] Hu X-L., Hou G-M., Zhang M-Q., Rong M-Z., Ruan W-H., Giannelis E. P.: A new nanocomposite polymer electrolyte based on poly(vinyl alcohol) incorporating hypergrafted nano-silica. Journal of Materials Chemistry, 22, 18961-18967 (2012).

DOI: $10.1039 /$ C2JM33156J

[4] Wang M. H., Ruan W. H., Huang Y. F., Ye L., Rong M. Z., Zhang M. Q.: A strategy for significant improvement of strength of semi-crystalline polymers with the aid of nanoparticles. Journal of Materials Chemistry, 22, 4592-4598 (2012).

DOI: $10.1039 / \mathrm{C} 2 \mathrm{JM} 16097 \mathrm{H}$

[5] Chen H., Zhang X., Zhang P., Zhang Z.: Facile approach in fabricating superhydrophobic $\mathrm{SiO}_{2}$ /polymer nanocomposite coating. Applied Surface Science, 261, 628632 (2012).

DOI: $10.1016 /$ j.apsusc. 2012.08.071

[6] Müller M. T., Krause B., Pötschke P.: A successful approach to disperse MWCNTs in polyethylene by melt mixing using polyethylene glycol as additive. Polymer, 53, 3079-3083 (2012).

DOI: $10.1016 /$ j.polymer.2012.05.041

[7] Tao F., Bonnaud L., Auhl D., Struth B., Dubois P., Bailly C.: Influence of shear-induced crystallization on the electrical conductivity of high density polyethylene carbon nanotube nanocomposites. Polymer, 53, 59095916 (2012).

DOI: $10.1016 /$ j.polymer.2012.10.026

[8] Liu T., Wood W., Li B., Lively B., Zhong W-H.: Effect of reinforcement on wear debris of carbon nanofiber/ high density polyethylene composites: Morphological study and quantitative analysis. Wear, 294-295, 326335 (2012). DOI: 10.1016/j.wear.2012.07.010

[9] Kohlman R. S., Joo J., Epstein A. J.: Physical properties of polymers handbook. American Institute of Physics, New York (1996).

[10] Trivedi D. C.: Handbook of organic conductive molecules and polymers. Wiley, New York (1997).

[11] Zhang L. J., Wan M. X., Wei Y.: Hollow polyaniline microspheres with conductive and fluorescent function. Macromolecular Rapid Communications, 27, 888-893 (2006).

DOI: $10.1002 /$ marc. 200600134

[12] He H., Zhu J., Tao N. J., Nagahara L. A., Amlani I., Tsui R.: A conducting polymer nanojunction switch. Journal of the American Chemical Society, 123, 77307731 (2001). DOI: $10.1021 / \mathrm{ja} 016264 \mathrm{i}$ 
[13] Grgur B. N., Ristić V., Gvozdenović M. M., Maksimović M. D., Jugović B. Z.: Polyaniline as possible anode materials for the lead acid batteries. Journal of Power Sources, 180, 635-640 (2008). DOI: 10.1016/j.jpowsour.2008.02.022

[14] Milczarek G.: Electrocatalytic thin films based on nordihydroguaiaretic acid-functionalyzed polyaniline. Reactive and Functional Polymers, 68, 1542-1548 (2008). DOI: 10.1016/j.reactfunctpolym.2008.08.006

[15] Choi H. J., Kim J. W., To K.: Electrorheological characteristics of semiconducting poly(aniline-co-o-ethoxyaniline) suspension. Polymer, 40, 2163-2166 (1999). DOI: 10.1016/S0032-3861(98)00418-2

[16] Kim J. W., Kim S. G., Choi H. J., Jhon M. S.: Synthesis and electrorheological properties of polyaniline$\mathrm{Na}^{+}$-montmorillonite suspensions. Macromolecular Rapid Communications, 20, 450-452 (1999). DOI: 10.1002/(SICI)1521-3927(19990801)20:8<450:: AID-MARC450>3.0.CO;2-N

[17] Klaysom C., Moon S-H., Ladewig B. P., Lu G. Q. M., Wang L.: The influence of inorganic filler particle size on composite ion-exchange membranes for desalination. The Journal of Physical Chemistry C, 115, 1512415132 (2011). DOI: $10.1021 / \mathrm{jp} 112157 \mathrm{z}$

[18] Huang J., Zhang K., Wang K., Xie Z., Ladewig B., Wang H.: Fabrication of polyethersulfone-mesoporous silica nanocomposite ultrafiltration membranes with antifouling properties. Journal of Membrane Science, 423-424, 362-370 (2012).

DOI: $10.1016 /$ j.memsci.2012.08.029

[19] Suzuki N., Kiba S., Yamauchi Y.: Low dielectric property of novel mesoporous silica/polymer composites using smart molecular caps: Theoretical calculation of air space encapsulated inside mesopores. Microporous and Mesoporous Materials, 138, 123-131 (2011). DOI: $10.1016 /$ j.micromeso.2010.09.020

[20] Suzuki N., Kiba S., Kamachi Y., Miyamoto N., Yamauchi Y.: Mesoporous silica as smart inorganic filler: Preparation of robust silicone rubber with low thermal expansion property. Journal of Materials Chemistry, 21, 5338-5344 (2011).

DOI: $10.1039 /$ C0JM03767B

[21] Romanes M. C., D’Souza N. A., Coutinho D., Balkus K. J., Scharf T. W.: Surface and subsurface characterization of epoxy-mesoporous silica composites to clarify tribological properties. Wear, 265, 88-96 (2008). DOI: 10.1016/j.wear.2007.08.022

[22] Huang K-Y., Weng C-J., Huang L-T., Cheng T-H., Wei Y., Yeh J-M.: Systematically comparative studies on the preparation and physical properties of PMMA-silica mesocomposite and nanocomposite membranes. Microporous and Mesoporous Materials, 131, 192203 (2010).

DOI: $10.1016 /$ j.micromeso.2009.12.022
[23] Weng C-J., Huang K-Y., Chiu W-L., Yeh J-M., Wei Y., Liu S-P., Hwang S-S.: A comparative study of the preparation and physical properties of polystyrene-silica mesocomposite and nanocomposite materials. Polymer International, 60, 1129-1135 (2011).

DOI: $10.1002 /$ pi.3052

[24] Wei Y., Jin D., Ding T., Shih W-H., Liu X., Cheng S. Z. D., Fu Q.: A non-surfactant templating route to mesoporous silica materials. Advanced Materials, 10, 313316 (1998).

DOI: 10.1002/(SICI)1521-4095(199803)10:4<313:: AID-ADMA313>3.0.CO;2-M

[25] Lowell S.: Introduction to powder surface area. Wiley, New York (1979).

[26] Cheng C-F., Cheng H-H., Cheng P-W., Lee Y-J.: Effect of reactive channel functional groups and nanoporosity of nanoscale mesoporous silica on properties of polyimide composite. Macromolecules, 39, 75837590 (2006).

DOI: $10.1021 / \mathrm{ma} 060990 \mathrm{u}$

[27] Ying J. Y., Mehnert C. P., Wong M. S.: Synthesis and applications of supramolecular-templated mesoporous materials. Angewandte Chemie International Edition, 38, 56-77 (1999).

DOI: 10.1002/(SICI)1521-3773(19990115)38:1/2<56:: AID-ANIE56>3.0.CO;2-E

[28] Corma A.: From microporous to mesoporous molecular sieve materials and their use in catalysis. Chemical Reviews, 97, 2373-2420 (1997).

DOI: $10.1021 / \mathrm{cr} 960406 \mathrm{n}$

[29] Barrett E. P., Joyner L. E., Halenda P. P.: The determination of pore volume and area distributions in porous substances. I. Computations from nitrogen isotherms. Journal of the American Chemical Society, 73, 373380 (1951).

DOI: $10.1021 / \mathrm{ja} 01145 \mathrm{a} 126$

[30] Cho M. S., Choi H. J., Ahn W-S.: Enhanced electrorheology of conducting polyaniline confined in MCM-41 channels. Langmuir, 20, 202-207 (2004). DOI: $10.1021 / 1 \mathrm{a} 035051 \mathrm{z}$

[31] Nishio E., Ikuta N., Hirashima T., Koga J.: PyrolysisGC/FT-IR analysis for silane coupling treatment of glass fibers. Applied Spectroscopy, 43, 1159-1164 (1989). DOI: $10.1366 / 0003702894203589$

[32] Subramani S., Cheong I. W., Kim J. H.: Synthesis and characterizations of silylated polyurethane from methyl ethyl ketoxime-blocked polyurethane dispersion. European Polymer Journal, 40, 2745-2755 (2004). DOI: 10.1016/j.eurpolymj.2004.07.018

[33] Subramani S., Lee J. M., Lee J-Y., Kim J. H.: Synthesis and properties of room temperature curable trimethoxysilane-terminated polyurethane and their dispersions. Polymers for Advanced Technologies, 18, 601609 (2007).

DOI: $10.1002 /$ pat. 860 
[34] Trchová M., Šeděnková I., Tobolková E., Stejskal J.: FTIR spectroscopic and conductivity study of the thermal degradation of polyaniline films. Polymer Degradation and Stability, 86, 179-185 (2004).

DOI: $10.1016 /$ j.polymdegradstab.2004.04.011

[35] Serrano D. P., Aguado J., Morales G., Rodríguez J. M., Peral A., Thommes M., Epping J. D., Chmelka B. F.: Molecular and meso- and macroscopic properties of hierarchical nanocrystalline ZSM-5 zeolite prepared by seed silanization. Chemistry of Materials, 21, 641-654 (2009).

DOI: $10.1021 / \mathrm{cm} 801951 \mathrm{a}$

[36] Weng S., Lin Z., Zhang Y., Chen L., Zhou J.: Facile synthesis of SBA-15/polyaniline nanocomposites with high electrochemical activity under neutral and acidic conditions. Reactive and Functional Polymers, 69, 130-136 (2009).

DOI: $10.1016 /$ j.reactfunctpolym.2008.12.001
[37] Feng X., Yang G., Liu Y., Hou W., Zhu J-J.: Synthesis of polyaniline/MCM-41 composite through surface polymerization of aniline. Journal of Applied Polymer Science, 101, 2088-2094 (2006).

DOI: $10.1002 /$ app. 23836

[38] Kaul P. B., Day K. A., Abramson A. R.: Application of the three omega method for the thermal conductivity measurement of polyaniline. Journal of Applied Physics, 101, 083507/1-083507/8 (2007).

DOI: $10.1063 / 1.2714650$

[39] Lu X., Nilsson O., Fricke J., Pekala R. W.: Thermal and electrical conductivity of monolithic carbon aerogels. Journal of Applied Physics, 73, 581-584 (1993). DOI: $10.1063 / 1.353367$ 